\title{
Tendencias educativas y socioeconómicas de los alumnos que cursan el último año de la Educación Media Superior en el municipio de Tepeji y su zona de influencia
}

\author{
Educational and socio-economic trends of students in the last year of Higher Middle Education \\ in the municipality of Tepeji and its influence area. \\ Ma. de Lourdes E. García-Vargas a*, Héctor D. Molina-Ruiz a , Jorge M. Hernández-Mendoza a \\ Magda G. Sánchez-Trujillo ${ }^{a}$
}

\begin{abstract}
:
Demand for higher education is increasing and the number of institutions responsible for preparing quality and relevant professionals is growing, so opening up new areas of knowledge that are intended to be offered at the Higher Level requires consider and analyze the characteristics of the regional environment and its area of influence

by pointing out on issues such as: expectations of the population segments to which new higher education services are directed, personal and family characteristics, intensity and desire to continue studying, economic possibilities to continue, the level of studies that it intends to continue, the program that it wants to study, educational institution in which it wishes to continue studying and your preference for choice. In this way, the present work managed to determine the socioeconomic factors and educational expectations of 753 students of Higher Middle Education belonging to the Tepeji del Río municipality, Hidalgo and its influence area, in order to make sound decisions for the proposed educational offer.
\end{abstract}

Keywords:

Higher Middle Education, socioeconomic study, educational trends

\begin{abstract}
Resumen:
La demanda de educación superior va en aumento y el número de instituciones encargadas de preparar profesionales de calidad y pertinentes va ascendiendo, por lo que para la apertura de nuevas áreas de conocimiento que se pretenden ofrecer en el Nivel Superior se requiere considerar y analizar las características del entorno regional y su área de influencia puntualizando en temas como: expectativas de los segmentos poblacionales a que se dirigen los nuevos servicios de educación superior, características personales y familiares, la intensidad y deseo de seguir estudiando, posibilidades económicas para continuar, el nivel de estudios que pretende continuar, programa que desea estudiar, institución educativa en la que desea continuar estudiando y su preferencia de elección. De esta forma, el presente trabajo logro determinar los factores socioeconómicos y las expectativas educativas de 753 alumnos de la Educación Media Superior pertenecientes al municipio de Tepeji del Río, Hidalgo y su área de influencia, a fin de tomar decisiones acertadas para la propuesta de oferta educativa.
\end{abstract}

\section{Palabras Clave:}

Educación Media Superior, estudio socioeconómico, tendencias educativas

\section{Introducción}

Frente a la cambiante sociedad mundial impulsada por el conocimiento, la educación superior mexicana requiere profundas transformaciones para la innovación y renovación de sus vínculos y manera de interactuar con la sociedad; por ello, las instituciones educativas deben asumir los retos y desafíos que le son planteados para alcanzar su desarrollo), como por ejemplo innovar sus métodos educativos centrados en los alumnos, para propiciar como base de su formación permanente que aprendan a aprender, a emprender y a ser, impulsar su creatividad e iniciativa, así como un espíritu crítico y un sentido de responsabilidad social del más alto nivel de calidad en el plano humanístico, cultural, técnico, profesional y científico [1]. Ampliar la cobertura de las escuelas mediante el incremento y diversificación de la oferta educativa presencial y virtual con programas

\footnotetext{
a Escuela Superior Tepeji del Rio, Ingeniería Industrial, Universidad Autónoma del Estado de Hidalgo, Av. del Maestro No.41, Col. Noxtongo 2a Sección, Tepeji del Rio, Hidalgo, México, C.P. 42855. Email: *maria_garcia10476@uaeh.edu.mx
} 
educativos más pertinentes, innovadores y flexibles que le permitan atender la creciente demanda de educación media superior y superior, así como la actualización de conocimientos durante toda la vida de sus egresados y del público en general. Asegurar la calidad y competitividad académica de su oferta educativa, brindando a los estudiantes servicios educativos de calidad que les proporcionen una sólida formación integral acorde a indicadores y estándares de la sociedad del conocimiento. Propiciar la equidad de acceso de los egresados del bachillerato a la Educación Superior (ES) mediante sistemas de admisión basados exclusivamente en el mérito y en la capacidad de los aspirantes, impulsando distintas vías de formación para facilitar el acceso a los grupos sociales desfavorecidos y a una mayor participación de la mujer [2].

Para ampliar su cobertura, mediante el incremento y diversificación de su oferta educativa presencial y virtual con programas educativos más pertinentes se considera vital el estudio de tendencias socioeconómicas y de expectativas educativas, seguir un lineamiento metodológico, y una investigación documental fundamentada en la consulta de archivos oficiales para establecer el marco contextual y teórico, revisar las necesidades de la población a través de un esquema de muestreo que converge en una investigación de campo utilizando un cuestionario como instrumento de recolección para obtener información sobre las características de las familias, las condiciones socioeconómicas de las mismas, así como los rasgos de los jóvenes entrevistados y expectativas respecto a su educación (formación profesional). En este trabajo se desarrollan los puntos considerados y se presentan los resultados y conclusiones logrando el objetivo general al determinar las tendencias educativas y socioeconómicas de los alumnos que cursan el último año de la Educación Media Superior para que las instituciones amplíen su cobertura mediante el incremento y diversificación de su oferta educativa a través de los objetivos específicos al reconocer las preferencias educativas de los alumnos en el proceso de incorporación al Nivel Superior.

Conocer si los alumnos cuentan con los servicios en la vivienda necesarios para realizar estudios superiores (un espacio digno en su casa para estudiar, servicio de Internet en su hogar, etc.).

\section{Método}

El estudio se realizó mediante una investigación documental y una investigación de campo con un enfoque cuantitativo, el alcance es descriptivo porque busca especificar propiedades y características importantes de cualquier fenómeno que se analice, así como las tendencias de una población o grupo objeto de estudio [3].

En el marco contextual el municipio de Tepeji del Río de Ocampo, Hidalgo colinda al norte con el estado de México, el municipio de Tula de Allende y Atotonilco de Tula; al este con el municipio de Atotonilco de Tula y el estado de México; al sur con el estado de México; al oeste con el estado de México. Es un importante centro industrial, que cuenta con 15 microindustrias, 2 industrias pequeñas, 15 medianas y 2 correspondientes a la categoría de gran industria. La principal producción de estas industrias es primordialmente textil, le siguen la de alimentos y bebidas, construcción, maquilas y material de acero, debido a su giro industrial, en este municipio se realiza un gran movimiento en materia comercial [4].

Actualmente en la Educación Media Superior (EMS) existen tres tipos de programas: el bachillerato general, cuyo propósito principal es preparar a los alumnos para ingresar a instituciones de educación superior; el profesional técnico, que proporciona una formación para el trabajo y el bivalente o bachillerato tecnológico que es una combinación de ambas. Los bachilleratos general y tecnológico se imparten bajo las modalidades de enseñanza abierta y educación a distancia. Asimismo, la opción técnica ofrece ya la posibilidad de ingresar a la Educación Superior y los planteles en donde se atiende a los alumnos de EMS pueden ser: federales, estatales, autónomos y privados [1]

La muestra se determinó considerando las instituciones que se encuentran dentro de un radio aproximado de 50 kilómetros a partir del punto centro del Municipio de Tepeji del Río, Hidalgo [5] (Figura 1) incluyendo municipios de los estados de Hidalgo y México, esto incluye tanto a municipios del estado de Hidalgo como a municipios colindantes pertenecientes al estado de México.

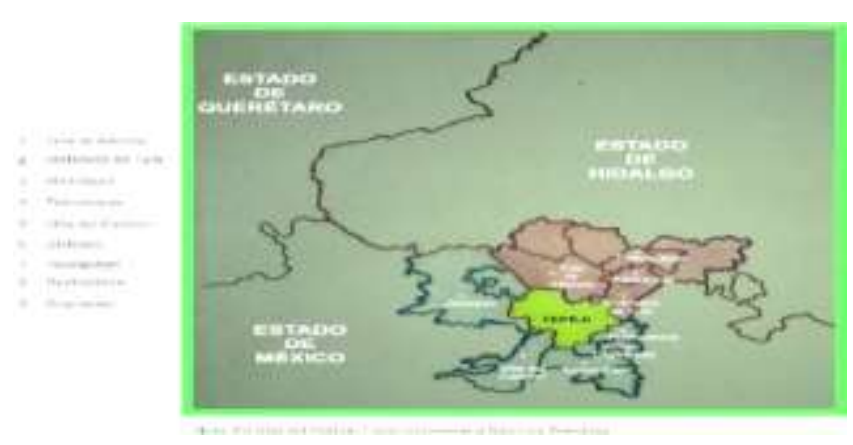

Figura 1. Mapa del municipio de Tepeji y su área de influencia

Mapa con la zona de influencia del municipio de Tepeji y los municipios considerados. La descripción detallada de las instituciones del Nivel Medio Superior involucradas en 
el estudio se describe y específica en el anexo número uno.

Se realizó una depuración de las bases de datos considerando las características establecidas para el estudio considerando 28 Instituciones de Educación Media Superior, definidas como la población a ser considerada.

Para cumplir con los objetivos específicos se aplicó un cuestionario a los alumnos que cursan la Educación Media Superior (EMS) que incluyen preguntas sobre las características de las familias, las condiciones socioeconómicas, los rasgos de los jóvenes entrevistados y expectativas respecto a su educación (formación profesional).

La investigación de campo comprendió las siguientes etapas: a) Diseño del instrumento de captación (cuestionario). b) Aplicación del cuestionario. c) Codificación, depuración, clasificación y análisis de la información. d) Supervisión durante todo el ciclo de captura de la información de manera aleatoria.

Unidad de análisis. Los participantes fueron 753 Hombres y mujeres que cursan los últimos grados de la EMS incluidos en la zona de influencia de la Escuela Superior Tepeji.

Muestreo. Se define bajo los criterios de inclusión y exclusión, dando mayor importancia a las instituciones más cercanas al punto en cuestión (Tepeji del Río, Hidalgo) e instituciones dispuestas a colaborar con el proyecto.

Método de recolección. Se generó la información por medio de un cuestionario que contiene las siguientes secciones: I. Datos del estudiante; II. Características de las familias; III. Condiciones socioeconómicas de las familias; IV. Características de las expectativas respecto a su educación superior.

Características del Instrumento de medición. Las características de este cuestionario son: Claridad: Se utilizó un lenguaje fácilmente asimilable por el entrevistado. Precisión: Se buscó definir bien las cuestiones para evitar ambigüedades. Duración limitada: Se redujo el tamaño del cuestionario lo máximo posible, evitando preguntas repetidas o excesivamente largas. Se aplicó una prueba piloto a 60 alumnos de manera aleatoria para detectar su consistencia, veracidad, fácil comprensión, se registra el tiempo se identifican reactivos donde los alumnos tenían duda. Levantamiento. El levantamiento del instrumento contó con 753 encuestas aplicadas en los distintos Institutos de la EMS de la Región Tepeji y su área de Influencia.

\section{Resultados}

La información contenida se analizó en el paquete Microsoft Office Excel para el desglose de tablas y gráficos a fin de dar respuesta a los objetivos planteados.

El instrumento recoge información sobre datos del estudiante, nombre del encuestado, sexo, edad en años cumplidos y la pregunta ¿Trabajas actualmente?

Características de los encuestados: En el estudio predomina el sexo femenino con un $55 \%$, el $72 \%$ tiene entre 17 y 20 años cumplidos y la mayoría no trabaja actualmente y los que trabajan se dedican principalmente al comercio, al campo, son músicos o meseros.

En el rubro características de las familias se pregunta: ¿En la actualidad vive con? Con base a la pregunta anterior, escriba la ocupación y el grado máximo de estudios alcanzado por sus padres o tutor. Anote el número actual de integrantes de su familia que conviven juntos, incluyendo padres, hermanos, tíos $u$ otros familiares.

El $76 \%$ de los alumnos encuestados viven con sus padres 573 alumnos, el $16 \%$ solo con la madre, el $2 \%$ solo con el padre. Por los resultados se consideran alumnos integrados en una familia convencional. La ocupación que domina en el padre es el de empleado, le sigue el obrero y por último el comerciante. La ocupación de la madre es en su mayoría ama de casa, le sigue el de empleada y por último el de comerciante. El grado máximo alcanzado por el padre es secundaria, le sigue la preparatoria y la licenciatura. El grado máximo de estudios alcanzado por la madre es la secundaria, en segundo lugar, el de preparatoria y por último la licenciatura. El grado máximo del tutor es la primaria, en segundo lugar, la secundaria y por último la preparatoria. Predominan las familias con cuatro integrantes, le sigue la familia conformada por 5 integrantes.

Condiciones socioeconómicas de las familias. ¿La casa donde habitas con tu familia es? ¿El tipo de localidad donde radicas es? ¿Quién paga tus estudios? ¿Tienes posibilidades económicas de seguir estudiando? Indica si el hogar que habitas cuenta con agua, luz, drenaje, gas, teléfono, internet.

El $87 \%$ cuenta con casa propia, solo el $7 \%$ renta y el $4.6 \%$ vive en casa prestada. El $59 \%$ tiene su vivienda en zona urbana, al $40.5 \%$ de los alumnos encuestados sus padres les pagan los estudios, cerca del $40 \%$ solamente el padre, el $90 \%$ refiere tener posibilidades económicas de seguir estudiando y el hogar del $65 \%$ cuenta con todos los servicios (luz, agua, drenaje, gas, teléfono, internet).

En el segmento características de los jóvenes entrevistados y sus expectativas respecto a su educación superior (factores de entorno) se pregunta:

Al terminar el bachillerato ¿deseas continuar estudiando? Si pretendes continuar estudiando ¿qué 
tipo de estudios prefieres? Selecciona el área del conocimiento que te interesa estudiar, señala la institución educativa a la que te gustaría ingresar, ¿Estarías dispuesto a cambiar de residencia por continuar tus estudios?

El $98 \%$ desea seguir estudiando, los estudios que prefieren son los de licenciatura e ingeniería, el área del conocimiento que les interesa estudiar es la de ciencias físico-matemáticas y de las ingenierías (ciencias exactas), le sigue el área de las ciencias de la salud y las ciencias sociales y humanidades. Prefieren como primera opción a la Universidad Autónoma del Estado de Hidalgo, como segunda opción a la UNAM y al Politécnico Nacional como tercera opción. Estas instituciones son preferidas porque cumplen con sus expectativas, calidad académica y cercanía. El $87 \%$ está dispuesto a cambiar de residencia.

\section{Conclusiones}

De acuerdo con el análisis de los datos obtenidos, en el presente estudio se logró determinar que, la tendencia de los alumnos que cursan el último año de la EMS es por la licenciatura e ingeniería, la primera área del conocimiento que les interesa estudiar es la de ciencias físico-matemáticas, la segunda es la de Ingenierías (ciencias exactas), la tercera es el área de las ciencias de la salud y por último el área de ciencias sociales y humanidades.

Prefieren la Universidad Autónoma del Estado de Hidalgo, le siguen la UNAM y la Universidad politécnica (politécnico Nacional) porque cumplen con sus expectativas, calidad académica y cercanía.

El $87 \%$ está dispuesto a cambiar de residencia. El grado de estudios alcanzado por sus padres es la secundaria, el trabajo del padre es empleado, obrero comerciante y de la madre es ama de casa, la mayoría vive en casa propia. Los alumnos cuentan con los servicios en la vivienda necesarios para realizar estudios superiores (un espacio digno en su casa para estudiar, servicio telefónico e internet en su hogar, etc.) y con posibilidades económicas para la continuación de estudios de Licenciatura e Ingeniería, al $87 \%$ le interesa continuar sus estudios de educación superior.

\section{Referencias}

[1] SEP (2010) Instituciones de Educación Media Superior, Disponible en: http://www.snie.sep.gob.mx/SNIESC/default.aspx., [Consulta 13 de noviembre de 2018].

[2] Plan de Desarrollo Institucional 2013-2019 http://www.uaeh.edu.mx/dgp/actPDI2013/ayuda/Actualizacion\%20de $\% 201 a \% 20$ planeacion.pdf

[3] Metodología de la Investigación. Hernández, R., Fernández, C. y Baptista, P. Sexta ed. USA;2014: 90-169.

[4] INEGI. Cuaderno estadístico municipal (2005). Disponible en https://www.inegi.org.mx/app/biblioteca/ficha.html?upc=70282500112 6.

[5] SEP - Hidalgo, Educación Media Superior (Distribución de matrícula por edad y grado (2008). Disponible en: http://intranet.ehidalgo.gob.mx/seph/fc08-09/index.htm, [Consulta 2 de febrero de 2018]. 
Anexo 1.- Instituciones educativas consideradas para el estudio.

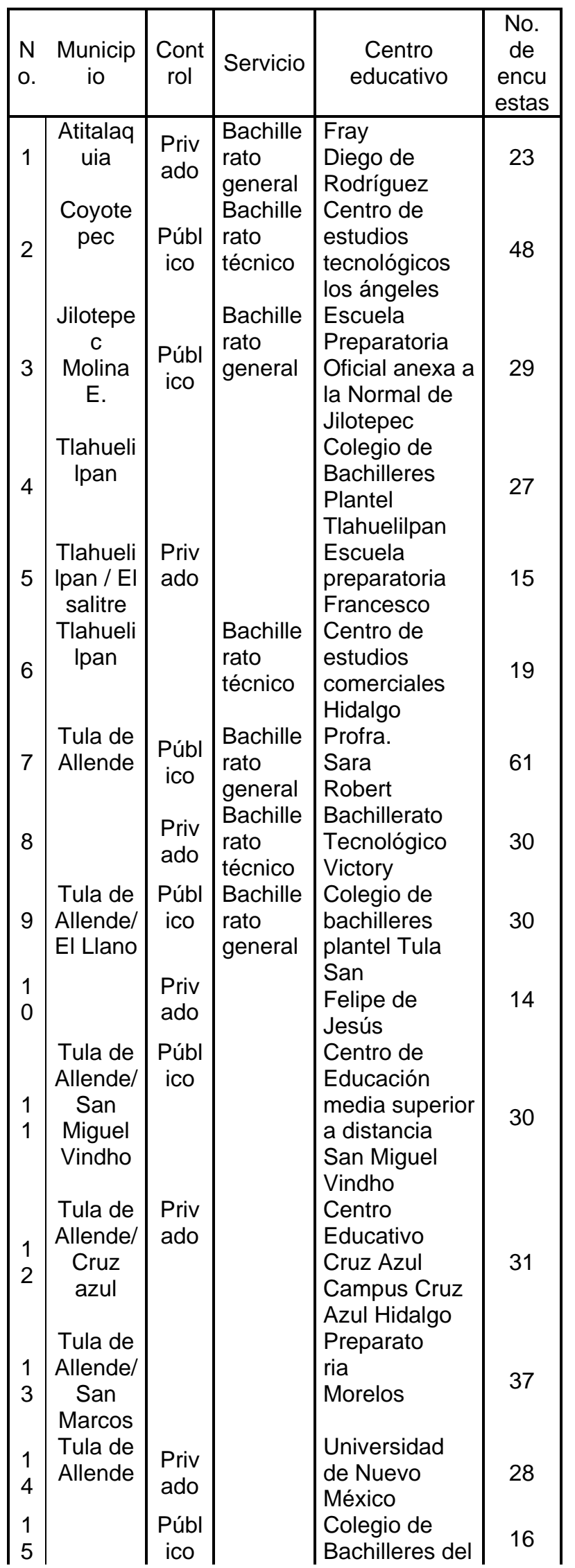

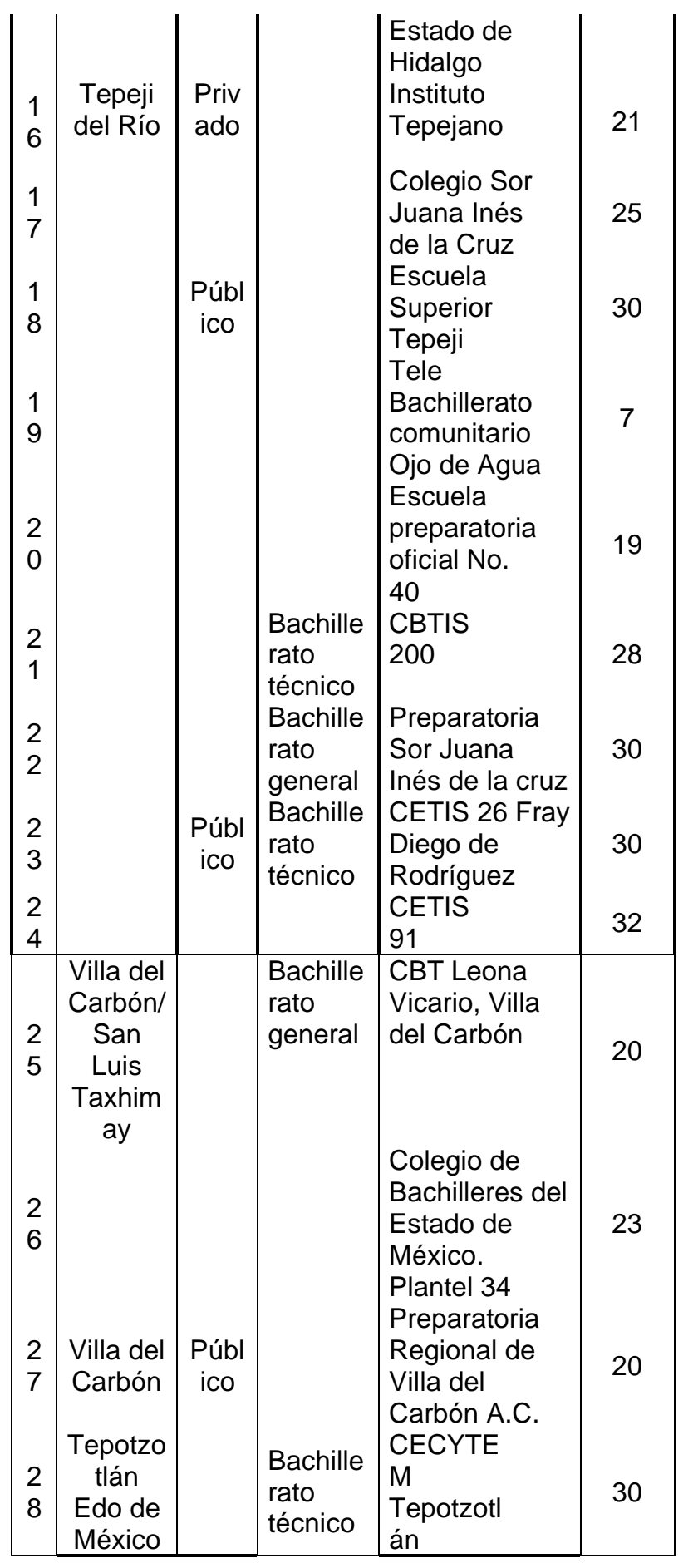

